\title{
How to fight brain cell suicide, and feel good at it
}

\author{
Adriano Aguzzi $^{1,2}$ and Gerhard F. Huber ${ }^{1}$ \\ ${ }^{1}$ Institute of Neuropathology, Schmelzbergstrasse 12, UniversitätsSpital Zürich, \\ $\mathrm{CH}-8091$ Zürich, Switzerland \\ 2 corresponding author, e-mail adriano@pathol.unizh.ch
}

Nobody knows why brain cells die - in most neurodegenerative diseases. The passionate fights between the proponents of alternative theories of neurodegeneration are a consequence of such lack of knowledge. Take Alzheimer's disease: is it due to the deposition of A- $\beta$ amyloid - or is it rather the cytoskeletal anomalies involving the tau protein which kill the crucial cortical and hippocampal neurons? And uncertainty can be even worse: contentious as the Alzheimer field may be, pathogenesis of prion diseases is even more of a black hole, with the theory of template-directed refolding competing against the seeding hypothesis (Aguzzi and Weissman, 1997), and a small but vociferous group of scientists who reject the protein-only hypothesis in any of its variations (Manuelidis et al, 1997).

Common to the two aforementioned diseases, and indeed to many others, is an irreversible destruction of neurons, which triggers all sorts of dismal neurological consequences. More often than not, death of neurons occurs by deliberate cell suicide. This is true even after well-defined 'environmental' events like axotomy, growth factor deprivation, ischaemia or oxidative stress - a recent and important discovery, which paves the way to promising strategies for therapeutical intervention. The reviews in this issue of Cell Death and Differentiation describe some of the pathways, genes and proteins involved in apoptotic cell death in the nervous system, and discuss some approaches to interfering with them.

Synthetic, unifying theories are always exciting, because they convey the feeling that we are getting closer to understanding the 'big scheme'. And precisely this has been happening in the last few years in the field of neurodegeneration. Hereditary and sporadic neurological diseases as diverse as Parkinson's, spinocerebellar ataxias, or Huntington's, are invariably associated with aggregates due to abnormal interactions of proteins. In this issue, Trojanowski and colleagues (1998) describe the role of synuclein in the formation of Lewy bodies, protein clumps found in the midbrain neurons of patients with Parkinson's disease. Although the actual function of synuclein is still not clear, mutations in its cognate gene seem to predispose to the formation of aggregates, in a way that is disturbingly similar to hereditary spongiform encephalopathies. Transgenic mouse models were pivotal in showing that filamentous inclusions (such as those occurring in human Lewy body dementia and Parkinson's disease) may kill neurons - although the mechanisms by which this occurs have yet to be understood. The ultimate result, of course, is disconnection of brain regions from each other (e.g. substantia nigra and striatum).

If intracellular protein aggregates are indeed the immediate cause of neuronal loss in these diseases, it would seem reasonable to attempt at interfering with their formation. If that proves trickier than predicted, one might think of identifying pathways downstream of protein aggregation, whose obstruction may protect neurons from apoptosis. One of the most promising implementations of the latter strategy involves utilisation of some of the most famous players in the apoptosis field: the proteins of the bcl-2 family (Sadoul, 1998). There is an obvious catch, of course. Bcl-2, when overexpressed, is a bona fide dominant oncogene which is commonly hyperactive in human malignant non-Hodgkin lymphomas. This observation can hardly be dismissed as an epiphenomenon, since bcl-2 transgenic mice also develop a lymphoproliferative disease secondary to extended survival of lymphocytes. And yet bcl-2 does not seem to bear neoplastic potential when expressed in CNS neurons - at least in mice. Transgenic animals that overexpress bcl-2 under control of the NSE promoter grow some 35\% more CNS neurons than their wild-type counterparts. While all these extra neurons are certainly of dubious value for fighting neurodegeneration, it turns out that the same transgenic animals enjoy drastically reduced apoptotic cell death of neurons after experimental brain injury. On the other hand, overexpression of bax (the pro-apoptotic counterpart of bcl2) kills sympathetic neurons even in the presence of NGF, while neurons of bax knockout mice are protected against axotomy-induced cell death.

There is no doubt that these observations are extremely exciting, and provide a clear pathway for discovery of new drugs apt at influencing the suicide programme of neuronal cells. But the beneficial effects of $\mathrm{Bcl}-2$ extend further than promotion of neuronal survival. Neuronal outgrowth and regenerative potential are not only depending on neurotrophic factors, but also on cellautonomous programmes within neurons. Even under optimized environmental conditions, axon regeneration does not take place in the postnatal CNS. While myelinassociated inhibitory glycoprotein have been incriminated for almost a decade as the main impediment to axon regeneration, we have now learnt that even after blocking (some) growth-inhibiting factors regeneration of CNS axons usually occurs only in a small proportion of neurons-therefore, something inside CNS neurons, in addition to environmental factors, might be dictating the limits to their regenerative potential.

Indeed, the ability of axonal regeneration can be regulated by bcl-2, as reviewed by Chen and Tonegawa (1998). Evidence from both neuronal organotypic cocultures using bcl-2 overexpressing CNS tissue, and from 
experiments in vivo, indicate that expression of bcl-2 in neurons correlates with the regenerative capacity of axons.

As exciting as all these data may be, it should not be forgotten that bcl-2 is a potent oncogene, and its carcinogenic properties pose formidable problems to any immediate clinical application. The oncogenic properties of bcl-2 are unlikely to be limited to lymphopoietic tissue, and indeed bcl-2 was shown to be highly expressed in various types of human astrocytomas and glioblastomas. While a role for bcl-2 in the histogenesis of the latter tumours has not been formally proven, this point needs to be pondered with care. That NSE-bcl-2 transgenic mice did not develop tumours is reassuring, especially since the tissue specificity of the NSE promoter is not restricted to neurons, but it certainly cannot be taken as a proof that the approach is totally safe.

The mammalian caspase family of cell death proteins is at least as important as the bcl-2 family for cell death and regeneration. There is good reason to believe that these proteins may represent yet another gratifying point of entry for tackling the problem of neurodegeneration. ICE (interleukin-1-beta converting enzyme), seems to be an important factor in neuronal apoptosis induction, especially under pathological conditions such as cerebral ischaemia. The role of ICE and other caspases in the pathogenesis of stroke, ALS, and Huntington's disease, is discussed in the review of Friedlander and Yuan (1998). Sure enough, ICE is upregulated in ischaemic neurons, and this is followed by apoptosis. Again, a series of transgenic experiments is shedding light on the causal relationships between these phenomena. In ICE knockout mice, brain injury after experimental cerebral ischaemia was drastically reduced. And, most excitingly, in a transgenic mouse model of amyotrophic lateral sclerosis, ICE seems to be activated and, maybe, to induce loss of motor neurons. Interleukin-1-beta, the product of ICE activation, is also supposed to have a function mediating neuronal cell death in ischaemic injuries. This suggests that inhibition of the Interleukin-1-beta pathway might be beneficial to treatment of stroke.

In summary, the surge of interest for neurodegenerative diseases among the cell death specialists is yielding some unexpected, extremely promising results. The review manuscripts in the present issue of Cell Death and Differentiation shows that the field of neuronal antiapoptosis has matured in an extremely short period of time. On a more personal note, we are grateful to the scientists who contributed these papers: for scientists resident in Switzerland, which has just escaped a plebiscite aimed at interdicting all experiments with transgenic mice, the achievements in the field of growth and death of nerve cells have offered an invaluable array of powerful arguments, and indeed many of the experiments described here were successfully used to explain to the general public the great usefulness of transgenic technologies to advance medical progress.

\section{References}

Aguzzi A and Weissmann C (1997) Prion research: the next frontiers. Nature 389: $795-798$

Chen DF and Tonegawa S (1998) Why do mature cns neurons of mammals fail to reestablish connections following injury - functions of bcl-2. Cell Death Differ. 5: $816-822$

Friedlander RM and Yuan J (1998) ICE, neuronal apoptosis and neurodegeneration. Cell Death Differ. 5: 823-831

Manuelidis L, Fritch Wand XiYG (1997) Evolution of a strain of CJD that induces BSElike plaques. Science 277: $94-98$

Sadoul R (1998) Bcl-2 family members in the development and degenerative pathologies of the nervous system. Cell Death Differ. 5: 805-815

Trojanowski JQ, Goedert M, Iwatsubo T and Lee VMY (1998) Fatal attractions: abnormal protein aggregation and neuron death in Parkinson's disease and Lewy body dementia. Cell Death Differ. 5: 832-837 